\title{
Political Incentives Towards Replacing Animal Testing in Nanotechnology?
}

Ursula G. Sauer

Scientific Consultancy - Animal Welfare, Neubiberg, Germany

\begin{abstract}
Summary
The Treaty of Lisbon requests the European Union and the Member States to pay full regard to animal welfare issues when implementing new policies. The present article discusses how these provisions are met in the emerging area of nanotechnology. Political action plans in Europe take into account animal welfare issues to some extent. Funding programmes promote the development of non-animal test methods, however only in the area of nanotoxicology and also here not sufficiently to "pay full regard" to preventing animal testing, let alone to bring about a paradigm change in toxicology or in biomedical research as such. Ethical deliberations on nanotechnology, which influence future policies, so far do not address animal welfare at all. Considering that risk assessment of nanoproducts is conceived as a key element to protect human dignity, ethical deliberations should address the choice of the underlying testing methods and call for basing nanomaterial safety testing upon the latest scientific-and ethically acceptable - technologies. Finally, public involvement in the debate on nanotechnology should take into account information on resulting animal experiments.
\end{abstract}

Keywords: nanotechnology, bioethics, non-animal testing strategies, European research funding programmes, political action plans

\section{Introduction}

Nanotechnology, an emerging technology considered to be a "key technology for the $21^{\text {st }}$ century", includes the development and practical applications of structures and devices on the scale of 1-100 $\mathrm{nm}$ in a large variety of research areas including medicine. The expectations raised by nanotechnology are overwhelming; the praise it receives sometimes even resembles religious awe. On its website regarding nanotechnological research, the European Union raves: "Nanotechnologies are something of a Holy Grail for many of today's research project teams, whether they are working on inanimate matter or living organisms. Manipulating arrangements between atoms to form nanosystems - with unique physical, chemical and biological properties - opens the door to applications which mark the beginning of a truly innovative technological era" ${ }^{\text {. }}$.

The European Commissioner for Science and Research, Janez Potočnik, expects nanotechnology to improve the lives of the European people: "Nanotechnology is an area which has highly promising prospects for turning fundamental research into successful innovations. Not only to boost the competitiveness of our industry but also to create new products that will make positive changes in the lives of our citizens, be it in medicine, environment, electronics or any other field" 2 .

In consequence of the expectations raised by nanotechnology, this area is currently receiving a lot of political attention and pub- lic funding. Animal welfare concerns regarding nanotechnology address the questions whether this new area of research is leading to an increase in animal experiments and, if so, how distressful such experiments are and how they can be prevented.

In pursuance of these questions, the Foundation Animalfree Research conducted a critical literature survey to obtain an overview on animal experiments and non-animal experiments in nanotechnology (Sauer, 2009). As of 2004, a continuous increase in scientific publications covering biomedical research in nanotechnology could be discerned. One-fourth of the publications related to studies conducted in vivo; whereas three-fourths of the publications depicted in vitro research. Nevertheless, also these estimations are disquieting from the point of view of animal welfare. Since nanotechnology is an emerging technology, the animal numbers could possibly indicate the beginning of a continuous increase in animal testing. Furthermore, a large proportion of the animal experiments evaluated were moderately and even severely distressful to the animals (Sauer, 2009).

Topics addressed by in vivo animal experiments in nanotechnology research were drug, gene, vaccine delivery with "nanocarriers", medical imaging technologies with nanoparticles, studies evaluating toxic effects of nanomaterials, issues of regenerative medicine and tissue engineering, as well as surgical procedures making use of nanotechnological innovations (Sauer, 2009). 
The results of the literature survey revealed that nanotechnology indeed gives rise to concern from the point of view of animal welfare, while at the same time also new non-animal technologies are being developed in the course of nanotechnological research.

The Treaty of Lisbon, on the other hand, which entered into force on 1 December 2009, calls upon the European Union and the Member States to pay full regard to the interests of animals as sentient beings when formulating new policies (Article 13 of the Treaty on the Functioning of the European Union): "In formulating and implementing the Union's agriculture, fisheries, transport, internal market, research and technological development and space policies, the Union and the Member States shall, since animals are sentient beings, pay full regard to the welfare requirements of animals, while respecting the legislative or administrative provisions and customs of the Member States relating in particular to religious rites, cultural traditions and regional heritage", (Treaty of Lisbon, 2008). Whereas this statement sets the framework for the European Union's attitude towards animals, the term "full regard" is imprecise and evasive. There are no legal definitions establishing when "full regard" has actually been met.

From the point of view of animal welfare, "paying full regard to animal welfare issues" should lead to the inclusion of animal welfare issues in political activities and ethical deliberations (since they in turn will become the frameworks for future political activities) and by making available sufficient funding and other incentives to ensure that the animal welfare goals can indeed be met. It is along these lines that the present article evaluates how political actions, scientific committee opinions and ethical deliberations regarding nanotechnology address animal welfare issues and to which extent research programmes promoting nanotechnology take them into consideration.

\section{Consideration of animal welfare issues in political action plans regarding nanotechnology}

\subsection{EU political actions regarding nanotechnology}

In June 2005, the European Commission adopted the EU Nanotechnology Action Plan 2005-2009 (Commission of the European Communities, 2005). In this action plan, the European Commission declares its commitment to promote nanotechnological research, to address the citizens' expectations and concerns regarding this new technology and to ensure the safety of products and techniques resulting from nanotechnology. Animal welfare issues are not specifically addressed in the Nanotechnology Action Plan.

On 6 September 2007, the European Commission adopted the first implementation report on the nanotechnology action plan (Commission of the European Communities, 2007). As regards research on safety aspects of nanotechnology, this progress report mentions the European Commission's Joint Research Centre (JRC) activities in developing in vitro and in silico methods: "The JRC, meanwhile, is focusing on the development and harmonization of methods for the characterization and toxicity testing of manufactured nanomaterials (e.g. particle size meas- urements, in vitro testing of a representative set of nanomaterials on critical cell lines); related studies on reference materials and dosimetry; studies on the applicability of computational methods for assessing nanoparticle properties, including toxicity; and database development." Apart from this statement, there is no direct or indirect reference to consideration of animal welfare issues in the first implementation report to the nanotechnology action plan.

Currently, specific legal requirements on the marketing or safety testing of nanomaterials do not exist. In February of 2008, the European Commission adopted a voluntary code of conduct on responsible nanotechnological research (Commission of the European Communities, 2008). The code of conduct lays down that nanotechnology and nanosciences (N\&N) should not harm or create a biological, physical or moral threat to people, animals, plants or the environment, at present or in the future (page 6). Key priority 4.1.13 (page 8) of the Code of Conduct grants the implementation of the 3Rs principle high priority: "Member States, $N \& N$ research funding bodies and organisations should encourage fields of $N \& N$ research with the broadest possible positive impact. A priority should be given to research aiming to protect the public and the environment, consumers or workers and aiming to reduce, refine or replace animal experimentation."

\subsection{Member State and Associated Countries political actions regarding nanotechnology}

The following overview depicts political action plans regarding nanotechnology in the German speaking EU Member States Germany and Austria as well as in the associated European country Switzerland.

\section{Germany}

In 2007, the German Federal Government adopted the "NanoInitiative - Action Plan 2010" (BMBF, 2007). Similarly to the aims of the EU action plan, the goals spelled out in the German action plan are to open up research areas, to improve general frameworks, to act responsibly and to inform the public. As regards animal welfare issues, the action plan confirms a dedication to avoiding animal testing in nanotechnological research: "The BMELV (Federal Ministry for Food, Agriculture and Consumer Protection) is especially concerned with ensuring that animal protection issues are taken into account right from the start and that alternative and complementary methods are used in place of animal testing wherever possible", (page 8).

In a status report on nanotechnology, the German Government declares its commitment to found nanotoxicological testing on non-animal test methods (BMBF, 2009, page 73): "The focus of the hazard analysis is on the field of in-vitro studies. First results of these studies indicate that at least the particle effects appearing in the lungs for a short time are predictable by in-vitro tests with alveolar macrophages, when a combination of different test parameters is evaluated. However, only the final test of all parameters can clarify whether and to which extent the developed in-vitro methodology is also suitable for the assessment of long-term particle effects." 


\section{Austria}

The Austrian government has adopted a so-called NANO Initiative, which is seen as a multi-annual "Funding Programme for Nanoscale Sciences and Nanotechnologies" coordinating nanotechnology-related measures on the national and regional levels ${ }^{3}$. The Austrian NANO Initiative has the following objectives: "Broadening the cooperation basis between science and industry, strengthening research competence in fields of application relevant to Austrian enterprises, accelerating technology transfer and increasing the economic utilisation of nanotechnology, improving access to know-how and to cooperation partners abroad, decreasing insecurities and information deficits with regard to health risks and environmental risks, establishing nanotechnology in the context of public perception of Austria as a research location, of science communication and of promoting young researchers" 4 . On the internet pages of the Austrian NANO Initiative reference to specific consideration of animal welfare issues cannot be discerned.

As a part of the NANO Initiative, the Austrian Government has set up a Nano-Forum, a national platform, which addresses science, enterprises, societies and associations, networks relevant to nanotechnology, finance companies and other strategic partners in Austria. The objectives of the Nano-Forum do not make reference to animal welfare issues ${ }^{5}$.

As an important part of the Austrian NANO Initiative, the Austrian Institute for Technological Impact Assessment (ITA, Institut für Technikfolgenabschätzung) is currently performing a project called "Nano Trust - Health and Environmental Risks of Nanotechnology" 6 . A workshop organised within the NanoTrust project on the "Risk Governance of Nanotechnologies - Possible Health Effects of Manufactured Nanomaterials" (24 September 2009) ${ }^{7}$ included presentations on in vitro test methods for nanotoxicological testing.

\section{Switzerland}

As regards Switzerland, on 9 April 2008, the Swiss Government adopted an action plan on the assessment and management of the risks of synthetic nanoparticle, which "is intended to show what endeavours are required in Switzerland in order to fill the gaps in our knowledge. It is based on a comparable EU Action Plan from June 2005" 8 .

The action plan's objectives address responsibility for the safety of nanomaterials and issues to promote the new technology (Swiss Federal Council, 2008, page 5): “Creating framework conditions for responsible handling of synthetic nanoparticles, creating scientific and methodological conditions to recognise and prevent possible harmful effects of synthetic nanomaterials on health and the environment, promoting public dialogue about the promise and risks of nanotechnology, better use of ex- isting promotional instruments for the development and market launch of sustainable applications of nanotechnology."

The Swiss Action Plan refers to both in vivo and in vitro test methods in its deliberations on research relating to the safety testing of nanomaterials, (page 14): "A particular need for research has been identified in the following areas: Health (toxicological in vitro and in vivo methods, toxicokinetics, clinical studies, particle translocation, metabolism, bioaccumulation and persistence, effects on organ systems, model systems)."

\section{Consideration of animal welfare issues in Scientific Committee opinions on the safety testing and risk assessment of nanomaterials}

Three scientific committees provide the European Commission with scientific advice on risk assessment issues - and therefore also possibly on animal testing issues: the Scientific Committee on Consumer Safety (SCCS, formerly Scientific Committee on Consumer Products SCCP), the Scientific Committee on Health and Environmental Risks (SCHER) and the Scientific Committee on Emerging and Newly Identified Health Risks (SCENIHR).

In 2007, the SCCP, which preceded the SCCS, published an opinion relating to nanomaterials in cosmetic products:

- SCCP Opinion on the safety of nanomaterials in cosmetic products of 18 December 2007 (SCCP, 2007).

In the light of the $7^{\text {th }}$ Amendment to the EU Cosmetics Directive, which imposes an animal testing ban on cosmetic ingredients and cosmetic products, SCCP (2007) recognizes the need for validated in vitro methods specifically developed or optimised for cosmetic ingredient application of nanomaterials.

Between 2006 and 2009, the SCENIHR published 4 opinions regarding risk assessment issues in nanotechnology:

- SCENIHR Opinion on the appropriateness of existing methodologies to assess the potential risks associated with engineered and adventitious products of nanotechnologies of 10 March $2006^{9}$,

- SCENIHR Opinion on the appropriateness of the risk assessment methodology in accordance with the technical guidance documents for new and existing substances for assessing the risks of nanomaterials of 21-22 June $2007^{10}$,

- SCENIHR Opinion on the scientific aspects of the existing and proposed definitions relating to products of nanoscience and nanotechnologies of 29 November $2007^{11}$,

- SCENIHR Opinion on risk assessment of products of nanotechnologies of 19 January 2009 (SCENIHR, 2009).

SCENIHR (2009) considers in vitro test methods to be applicable as screening tests (page 13): "Currently, in vitro assays are use-

\footnotetext{
3 http://www.bmvit.gv.at/innovation/iktnano/nano.html4 http://cordis.europa.eu/nanotechnology/

4 http://www.nanoinitiative.at/evo/web/nano/390_EN.53D604136413034

5 http://www.nanoinitiative.at/evo/web/nano/378_EN.0

6 http://www.bmvit.gv.at/innovation/iktnano/nanotrust.html

7 http://nanotrust.ac.at/nano09/

8 http://www.bafu.admin.ch/chemikalien/01389/01393/01394/index.html?lang=en

$9 \mathrm{http}: / /$ ec.europa.eu/health/ph_risk/committees/04_scenihr/docs/scenihr_o_003b.pdf

$10 \mathrm{http}: / /$ ec.europa.eu/health/ph_risk/committees/04 scenihr/docs/scenihr_o_010.pdf

11 http://ec.europa.eu/health/ph_risk/committees/04_scenihr/docs/scenihr_o_012.pdf
} 
ful for screening purposes and may provide valuable insights into the underlying mechanisms of adverse effects. However, in vitro assays have their limitations, especially in relation to evaluation of a possible risk for humans and the environment. Therefore, at present, in vivo assays are still needed for risk assessment."

The SCENIHR backs up this estimation of the scientific potential of non-animal test methods by reference to publications by Warheit et al. (2007), Sayes et al. (2007), ECETOC (2006) and Oberdörster et al. (2005). Warheit and co-authors considered two in vitro test methods in their evaluation, the Ames bacterial reverse mutation test and an in vitro chromosomal aberration test with Chinese Hamster Ovary cells. The SCENIHR questions whether the nanomaterials used were actually able to enter the bacterial cells. Sayes and co-authors used three different in vitro cultures for determining in vitro pulmonary toxicity: cultures of rat L2 lung epithelial cells, primary alveolar macrophages collected from unexposed rats by bronchoalveolar lavage and cell co-cultures with both of these cell types. As SCENIHR (2009) report, the "toxicity end points showed little correlation between in vitro and in vivo measurements for inhalation toxicity profiles". Due to these observations, the SCENIHR questions the relevance of in vitro test methods for nanomaterial risk assessment.

Oberdörster et al. (2005) sum up the results of an expert working group convened by the International Life Sciences Institute Research Foundation/Risk Science Institute with the aim to develop a screening strategy for the hazard identification of engineered nanomaterials. The report presents "the elements of a screening strategy rather than a detailed testing protocol" (accentuation by Oberdörster). The three key elements of the presented toxicity screening strategy are: physicochemical characteristics, cellular and non-cellular in vitro assays, and in vivo assays. Nanotoxicological literature evaluated by Oberdörster and co-authors demonstrated comparability of in vivo and in vitro test results: "Tissue and cell culture analysis have also supported the physiological response seen in whole animal models and yielded data pointing to an increased incidence of oxidative stress, inflammatory cytokine production, and apoptosis in response to exposure to ultrafine particles." Thus, the expert working group started out by developing a screening strategy from the beginning, with this strategy including both in vitro and in vivo test methods. Oberdörster et al. (2005) consider in vitro methods to be an "important adjunct to in vivo studies". Like Sayes et al. (2007), they point to the "lack of validation against in vivo adverse effects" of in vitro methods.

Similarly to Oberdörster and co-authors, ECETOC (2006) suggests a tiered testing strategy for nanomaterial safety testing: "With regard to a general testing approach for human health hazard evaluation of nanoparticles the following was concluded: A first step would include a prioritisation-type in vitro screening strategy to assess the possible reactivity, biomarkers of inflammation and cellular uptake of nanoparticles. This strategy would determine likely potency but should ultimately be validated using in vivo techniques."

On behalf of the results of the mentioned publications, SCENIHR (2009) comes to the conclusion that in vitro assays require validation to become applicable for risk assessment, whereas in vivo test methods are considered to be useful due to the "experience" gained with them: "So, they (in vitro assays) may be used for assessing the possible reactivity, inflammatory potential and cellular uptake of nanoparticles. However, to be applicable in risk assessment, these assays need to be validated and their relevance for in vivo hazard identification needs to be demonstrated. It should be noted that the in vivo assays as described in the various OECD guidelines are not validated for nanomaterials either. However, the experience gained in the testing of chemicals with these assays indicates that they can be used for the detection of some potential human and ecological hazards."

For scientific reasons this conclusion should be questioned. It goes without saying that assays need to be validated and their relevance for in vivo - i.e. human - hazard identification demonstrated in order to become applicable for risk assessment. Likewise, the SCENIHR's observation that also in vivo assays have not yet been validated is correct. However, there are no scientific reasons to support the assumption that the experience gained in the testing of chemicals with in vivo assays indicates that they can be used for the detection of some potential hazards of nanomaterials. "Learning from experience may be nothing more than learning to make the same mistakes with increasing confidence", (Skrabanek and McCormick, 1989).

Experience can only supplement validation as a prerequisite for the sound application of any test method. Moreover, experience is not an issue distinguishing in vivo from in vitro tests. Experts in the area of alternative methods should be involved in the task to develop non-animal testing strategies for nanomaterial safety testing.

The scientific studies cited by the SCENIHR in their evaluation of the applicability of in vitro test methods for nanomaterial testing performed conventional in vitro assays, such as the Ames test, the in vitro chromosomal aberration test with $\mathrm{CHO}$ cells and in vitro cell culture assays with rat lung epithelial cells and rat alveolar macrophages. However, a comprehensive nonanimal testing strategy for nanomaterial testing should include in vitro assays making use of human cell cultures, since human cells more closely mimic the situation in humans.

Additionally, as regards the in vitro methodologies used by Sayes and co-authors, the nanomaterials were suspended in media for exposure to the cells. Accordingly, Sayes et al. report on various degrees of agglomeration of the nanoparticles in the suspension. Thus, it is unclear whether and in which concentration the substances actually reached the cells in their nanoparticulate form. Technologies for applying nanomaterials to in vitro cultures, however, should ensure consistent controlled exposure to the actual nanoparticles at a known concentration and known particle size. This has been recognized as a crucial issue decisive for the outcome of studies testing nanomaterials. Accordingly, dose-controlled systems have been developed to ensure consistent cell exposure to aerosolized particles (Lenz et al., 2009). Furthermore, application of the nanoparticulate test substances suspended in culture medium by no means resembles the physiological situation in the lung. Test methods specifically developed for pulmonary nanomaterial testing should be selected, such as co-culture models with human cells simulating the air-liquid interface (Brandenberger et al., 2009). 
Finally, one of the endpoints tested by Sayes et al. (2007) was MTT Reduction. However this detection method has been recognized as being badly suited for nanomaterial testing. In colorimetric assays, nanomaterials can interfere with the indicator dyes thereby seriously hampering the validity of the test results (Wahl et al., 2008; Gellein et al., 2009). Regarding the time points selected by Sayes et al. (2007) to determine the endpoints in vivo (acute versus chronic) and in vitro (at 1, 4, 24, $48 \mathrm{~h}$ ), it can be questioned whether they are adequate for a comprehensive comparison of the in vivo and in vitro results.

In consequence, the scientific studies discussed by the SCENIHR do not appear conclusive in determining the scientific possibilities of in vitro test methods for nanomaterial safety testing. While it goes undisputed that further research is required to develop and validate non-animal testing strategies for nanomaterial testing, in the meantime, with the validation of any test method for nanomaterial testing pending, all nanotoxicological testing can only serve screening purposes - be it performed in vivo or in vitro, just as was pointed out by Oberdörster and co-authors.

\section{Consideration of animal welfare issues in ethical deliberations regarding nanotechnology}

\subsection{Opinion of the European Group on Ethics in Science and New Technologies on ethical aspects of nanomedicine}

The European Group on Ethics in Science and New Technologies (EGE) has the task to examine ethical questions arising from science and new technologies and on this basis to issue opinions to the European Commission in connection with the preparation and implementation of Community legislation or policies.

In 2007, the EGE published an opinion on ethical aspects of nanomedicine (EGE, 2007). A key issue discussed in this opinion is the protection of human dignity as a fundamental shared value of the European society. According to the EGE, nanomedicine raises issues concerning the protection of this fundamental value: "The interests of science are legitimate and justified insofar as they are compatible with human dignity and human rights."

Amongst other issues, such as human enhancement due to nanomedical devices, the costs of nanomedical therapies leading to societal imbalances or privacy protection issues (see Box 1, next page), the EGE discusses the importance of risk assessment in ensuring human dignity and points to the ethical problems of dealing with knowledge gaps when assessing the risks of nanomaterials: "Risk assessment is conceived not only as a technical element for the safe governance of nanotechnology but rather as a factor conducive to the protection of the human dignity and autonomy of the persons directly (medical applications) or indirectly (exposure to free nanoparticles) involved, as well as the protection of the environment." - "Product liability legislation addresses many of the problems that may be associated with the new materials, but as the risks are not readily assessed and assessable, liability based on negligence and lack of knowledge becomes a serious ethical problem."

As regards consideration of animal welfare issues, the EGE confirms adherence to the 3Rs principle: "The Group considers it essential that reliable and cost-effective systems for toxicology screening of nanomaterials are developed. The required animal testing should strictly follow the $3 R$ principles (Refinement, Reduction, and Replacement)." Nevertheless, the EGE seems to consider animal tests as the gold standard for safety evaluations and even calls for the development of animal tests for nanomaterial safety testing: "As far as nanomedicine is concerned, the risk assessment issues refer to possible health effects in terms of toxicity for the patients involved. For example, how do we check that, because of their greater capacity to pass through biological systems, nanodevices designed for drugs delivery would not induce negative side effects for the patients? Or how can we devise valid animal testing models to monitor such side effects?"

\subsection{EU projects relating to ethical aspects of nanotechnology}

Starting with the $5^{\text {th }}$ EU Research Framework Programme, the European Union has been funding projects, which deal with ethical issues of this emerging technology. In October 2008, the Directorate General Research of the European Commission published a report on EU projects on ethical, legal and social aspects (ELSA) of nanotechnology. These projects comprise a broad range of topics, such as privacy issues, acceptance of the new technology, human health issues, accessibility to the new technology, and issues of company liability (Hullmann, 2008). The ELSA Report presents a total of 27 EU funded projects dealing with ethical aspects of nanotechnology, 2 from the $5^{\text {th }} \mathrm{Re}$ search Framework Programme, 20 from the 6th and 5 from the $7^{\text {th }}$ Research Framework Programme. Details of these projects are summarized in Annex 1.

As a part of the survey at hand, the websites of the mentioned EU ELSA projects were evaluated as to their consideration of animal welfare issues. None of the ELSA projects reviewed explicitly included animal welfare issues in their ethical deliberations on the impact of nanotechnology (see Annex 1).

Instead, one of the projects relating to the risk assessment of nanoparticles, NANOSAFE2 (see project No. 2 in Annex 2), did refer to ethical problems relating to animal experiments: "Because in-vivo experiments, using animal models, are expensive, slow and ethically questionable, there is a strong demand for a low-cost high-throughput in-vitro assay without reducing the efficiency and reliability of the risk assessment."

\section{Consideration of animal welfare issues in EU research funding programmes regarding nanotechnology}

\subsection{General figures on EU funding in nanotechnology}

EU research programmes are considered to be "main initiatives" for the implementation of the European Action Plan on Nanotechnology (Aguar and Nicholas, 2008). In consequence, the $5^{\text {th }}, 6^{\text {th }}$ and $7^{\text {th }}$ EU Research Framework Programmes have dedicated significant funding to nanotechnology related research. During the $6^{\text {th }}$ Research Framework Programme alone, a total of $550 \mathrm{EU}$ projects on nanotechnology were funded 


\section{Box 1 - Ethical aspects of nanomedicine (EGE, 2007)}

Societal issues: Access to nanomedical developments from an individual perspective. Who can afford nanomedicine? Is nanomedicine leading to a two-class citizenship? Apart from the social question of discrimination and injustice, how do individuals cope with the gap between availability in principle and non-availability in their concrete lives?

Medical vs. non-medical uses - therapy vs. enhancement: New nanomedical tools can be used not only to transgress the border between medical and non-medical uses but also to open the door to ethically problematic enhancements. This raises questions not only for the state but also for the individual: how can we preserve the plurality of life-styles and avoid the transformation of the medical system into a mere service system for whatever desire individuals may have?

Informed consent: Consent may not be too difficult to obtain - but when is it informed? And when is it free? How should one deal with medical information, considering the knowledge gaps and uncertainties, and with predictive information where more extensive information becomes available much more rapidly than before?

Diagnostic complexity and increased personal responsibility: This trend in nanomedicine reinforces tendencies in health care where the improved precision, the increasing number of options and the speed of the diagnostics will on the one hand enhance personal freedom, at least theoretically. But it can also, on the other hand, create anxiety by increasing individual responsibility for the choices made. This may lead to a shift of responsibility between doctors and patients so that the responsibility of patients for their health will increase. If the most enthusiastic advocates of nanomedicine are right, it may also affect the very concepts of health and disease.

Third party uses - insurance companies \& employers, the individual and the state: How can privacy be protected, when more and more information can be used for surveillance rather than only for medical reasons? Where can the line be drawn between useful data storage within the medical context and non-medical data storage? What strategies are implemented to protect the individual's privacy in both contexts?

Societal dialogue: Public participation is of vital concern in democratic states. A societal dialogue should include ques- tions about who draws the lines between what is allowed, acceptable, and what is not; and who overviews those who draw the lines.

Military use of nanotechnology: A new generation of weapons could be created with nanotechnologies that could have disastrous consequences for health and the environment. This research clearly raises concerns about its potential impact on safety and human welfare.

Anthropological questions - changing the human condition: Nano-scale implants and devices may have an impact on autonomy, integrity, self-identity and freedom. In particular, what are the implications of the "man/machine" distinction, and in the perception of it, on a social level? How do our concepts of human beings change?

Research prioritisation: Research funding affects research in nanomedicine, and emerging research in nanomedicine will affect research funding. Research funding in general raises ethical issues concerning the criteria used in priority setting.

The temptation of exaggerating benefits ("hype") should also be considered. The competition for research funds may, with the assistance of media and science fiction writers, contribute to creating nanomedical hype with regard to the curability of all diseases.

Further ethical aspects of nanotechnology are discussed in the NanoBio-Raise Briefing Paper on Societal and Ethical Issues in Nanobiotechnology': Nanotechnologies have been especially prone to the current zeitgeist of exaggeration. Futurologists have made grandiose claims for their potential to transform our lives, that all will be inevitable and wonderful, asserting certainty about outcomes, which the writers cannot know.

As regards selection of scientific topics, the NanoBio-RAISE Briefing Paper on Nanomedicine ${ }^{2}$ notes: Nanomedicine aims at lowering mortality in advanced countries where mortality already is low. Regarding the expectations raised by biomedical research, the example of cancer research is discussed:

The 2004 presentation of the cancer nanotechnology initiative in the United States revolves around the goal of "eliminating death and suffering from cancer by 2015 ".

1 http://files.nanobio-raise.org/Downloads/BPethics.pdf - see Annex 1 for more information on the EU NanoBioRaise Project

$2 \mathrm{http}: / /$ files.nanobio-raise.org/Downloads/Nanomedicine-fullweb.pdf - see Annex 1 for more information on the EU NanoBioRaise Project 
(Commission of the European Communities, 2007). Being "main initiatives" for implementing the Action Plan, research programmes should also be key elements in "paying full regard to the welfare requirements of animals" as requested by the Treaty of Lisbon.

As Hullmann (2008) depicts, in the $5^{\text {th }}$ Framework Programme (1998-2002), the estimated funding level of nanotechnology was about $45 €$ million per year, with the overall budget being $14.96 €$ billion. Also according to Hullmann (2008), the 6th Framework Programme (2002-2006) contained "a strong focus on nanotechnology". Out of a total proposed funding of $17.5 €$ billion, $1.3 €$ billion has been devoted to the priority thematic area of research on nanotechnology, knowledge-based materials and new industrial processes.

For the $6^{\text {th }}$ Framework Programme, Hullmann counted 20 projects relating to ELSA and governance of nanotechnology, most of them being specific support actions or coordination actions. According to Hullmann, some of these projects are directly focused on nanotechnology, others treat nanotechnology as one of several case studies, and others in turn deal with general issues that can be applied to nanotechnology. Regarding the calculation of "nanotechnology"-specific funding of those projects, which do not address nanotechnology issues exclusively, Hullmann reports that while the overall EC funding of these projects amounts to 15.85 Mio. $€$, the "nano share" has been calculated by deducing the share, which is not directly relevant for nanotechnology. Doing this, an amount of 8.85 Mio. $€$, specifically aiming at ELSA and governance of nanotechnology, remains.

Finally, regarding the $7^{\text {th }}$ Framework Programme (20072013), Hullmann (2008) recognizes a considerable increase of the budget foreseen for nanotechnology forming part of Theme Four of the Framework Programme "Nanosciences, nanotechnologies, materials and new production technologies - NMP" within the "Cooperation" programme. According to Hullmann, the total budget of FP7 is $50.52 €$ billion, with $3.47 €$ billion for the theme "NMP".

In summing up the mentioned figures, the European Union is dedicating a total of approximately 5 billion $€$ to nanotechnology related research over the timeframe of 14 years, i.e. from 1998 until 2013.

Private and national funding is also made available to promote nanotechnology research. In Europe, national funding is estimated to make up approximately two thirds of the total public funding: "Over its lifetime, FP6 accounted for almost a third of total public expenditure in Europe for N\&N. Global expenditure in $N \& N$, both public and private, in the period 2004-06 was around EUR 24 billion. Europe accounts for more than a quarter of this worldwide total, with the EC funding directly accounting for 5-6\%" (Commission of the European Communities, 2007).

\subsection{EU funding of projects on the possible health, safety and environmental impacts of nanoparticles} - in vitro and in vivo test methods

How much of the total amount of funding in nanotechnology is dedicated to in vitro research and to research aiming at avoiding animal testing? The literature survey by Sauer (2009) revealed incentives to replace animal testing in nanotechnology in the area of risk assessment, but not in the area of fundamental biomedical research, such as nanomedicine. Likewise, reports on test methods performed for the efficacy testing of nanotechnological products and devices are not yet available. Also in the course of the survey at hand, no projects aiming at the development of non-animal test methods for nanomedical fundamental or applied research could be discerned. As regards projects in the risk assessment of nanomaterials, Aguar and Nicholas (2008) present an overview of past and ongoing research projects relating to possible impacts on the human health or environmental safety of nanoparticles that were funded by the EU Framework Programmes (FPs), by EU Member States, Candidate Countries and Countries associated to FP6 or FP7. "Being the first of its kind, this compilation has information of 106 projects, 14 of them are from the FPs which give around 32 million $€$ in grants. The others 92 projects are from the $E U$ Members States which spend around 47 million $€$ in grants. This makes a total of some 79 million $€$. This information may well be incomplete."

Part 2 of the Annex presents a list of 14 EU funded projects relating to the human health risk assessment of nanoparticles. Of these, research projects with the main goal to develop nonanimal test methods in nanotechnology are the FP6 projects CellNanoTox (Cellular Interaction and Toxicology with Engineered Nanoparticles) and DIPNA (Development of an Integrated Platform for Nanoparticle Analysis) and the FP7 project NANOtest (Development of alternative testing strategies for the assessment of the toxicological profile of nanoparticles used in medical diagnostics). Further research projects (e.g. Nanosafe2, NANOSH, PARTICLE-RISK, ENPRA, NANORETOX) also cover research on in vitro toxicological test methods, however do not address the development of non-animal test methods as main objective of the project (see Annex 2).

The funding granted to those projects directly aiming at developing non-animal test methods for nanomaterial toxicological testing amounts to $2.6 \mathrm{Mio}$ € for the project CellNanoTox, 2.8 Mio. $€$ for DIPNA, and 3.0 Mio. $€$ for the project NANOtest (Aguar \& Nicholas, 2008) - a total amount of approximately 9 Mio. $€$.

Over the last 20 years, the European Union's total financial contribution to the implementation of the 3Rs principle in general, regardless of its relation to the specific area of nanotechnology, amounted to some 200 Mio. $€$ (European Commission - European Research Area, 2009).

\section{Challenges and opportunities of nanotechnology from the point of view of animal welfare}

The Treaty of Lisbon obliges the European Union and the EU Member States to pay full regard to the welfare requirements of animals when implementing research and technological development policies. Nanotechnology is such a research area. Its political framework is currently under development. The survey at hand evaluates how and to which extent European political activities, 
ethical deliberations and public research programmes relating to nanotechnology take into account animal welfare issues.

As could be shown, the EU and national political action plans supporting nanotechnology and related initiatives do take into account animal welfare issues to some extent, at least by reference to the 3 Rs principle or indirectly by consideration of in vitro toxicity testing methods. However, the subsequent activities implementing the EU action plan, i.e. research funding programmes, scientific committee opinions and ethical deliberations, currently do not go far enough to pay full regard to animal welfare issues.

Research funding programmes relating to nanotechnology, considered to be "main initiatives" for implementing the nanotechnology action plan, promote the development of non-animal test methods, at least in the area of nanotoxicology and risk assessment of nanoparticles. In nanomedicine, no research projects could be discerned aiming at developing non-animal testing strategies, neither for fundamental research purposes nor for testing the efficacy of products or devices. In total, the amount of EU funding provided for non-animal test method development in nanotechnology, does not seem sufficient to pay "full regard to animal welfare issues".

So far, the European Union has made available approximately 9 Mio. $€$ to specifically fund the development of non-animal test methods for nanomaterial testing - with additional funding for projects which include the development of non-animal test methods amongst other project objectives. The development of non-animal test methods in general has been receiving EU funding of 200 Mio. $€$ over a time span of 20 years. In comparison, the entire key subject of nanotechnology is receiving a sum 25 times higher - and that over a shorter period of time: approximately $5 €$ billion EU funding over a time span of 14 years (Aguar and Nicholas, 2008).

In order for the development and validation of non-animal test method to also become a key subject, thereby paying full regard to animal welfare issues, its funding in nanotoxicology - and also nanomedicine - requires considerable adjustment. This request also meets the provisions of Article 23(1) of the European Directive on the Protection of Laboratory Animals 86/609/EEC, which explicitly calls upon the European Community and its Member States to encourage research into the development and validation of alternative techniques. It remains to be hypothesized how far the development and validation of non-animal test methods could proceed if this area would receive $5 €$ billion of EU funding over the time span of one and a half decades. Since the budget of the ongoing EU Research Framework Programme, which will continue until 2013, is set, at least projects aiming at developing or performing in vivo test methods should no longer be promoted under FP7 so that the respective funding will fully be made available for non-animal test method development.

Ethical deliberations on nanotechnology so far do not seem to take into consideration animal welfare issues at all. Considering that ethical discourses are likely to influence future political actions and public views, their impact on the future development of nanotechnology policies and regulations is evident. It is questionable whether nanotechnology regulations and policies can meet the provisions of the Treaty of Lisbon to pay full regard to the welfare requirements of animals if the underlying ethical deliberations on nanotechnology do not include animal welfare issues. Which aspects should ethical deliberations on nanotechnology include in order to comply with the animal welfare provisions of the Treaty of Lisbon?

Whereas the current nanotechnology "hype" is producing unrealistic expectations and possibly unethical products, the pain, suffering and distress of the animals involved in nanotechnological research is real.

A number of reports point to the "hype" associated with nanotechnology and question whether many of the expectations raised by nanotechnology will be met at all. Similarly, a variety of serious ethical concerns are being spelled out in regard to a number of nanotechnological products, concerns which might even lead to the proscription or prohibition of such products. In the meantime, however, animal experiments are being performed in nanotechnology, inevitably leading to pain, suffering and distress inflicted upon the animals involved.

The likelihood of achieving a scientific goal must be addressed in any cost-benefit-analysis of an animal experiment, in which the likely scientific benefit is balanced against the inevitable distress of the animals. From the point of view of animal welfare, it is unethical to perform distressful animal experiments, if the probability of achieving the goals pursued is questionable in the first place. Likewise, animal experiments should not be permissible for the development of products that raise serious ethical concerns. In such scientific areas, research involving sentient animals should be discontinued at least until the ethical debates have advanced to a stage that there is wide agreement as to which products are deemed acceptable.

Since nanotechnology safety testing requires new test methods and new test batteries, these should be based upon the latest scientific technologies instead of falling back on outdated unreliable animal test methods.

So far, validated test methods for nanomaterial testing do not exist. The safety testing of nanotechnological products requires new test methods and new test batteries. It would not be stateof-the-art to found such new testing strategies on animal tests. The National Academy of Sciences (2007) points to the scientific deficiencies of animal test methods: "Using the results of animal tests to predict human health effects involves a number of assumptions and extrapolations that remain controversial. Test animals are often exposed to higher doses than would be expected for typical human exposures, requiring assumptions about effects at lower doses or exposures. Test animals are typically observed for overt signs of adverse health effects which provide little information about biological changes leading to such changes leading to such health effects. Often controversial uncertainty factors must be applied to account for differences between test animals and humans. Finally, use of animals in testing is expensive and time consuming, and it sometimes raises ethical issues."

Accordingly, the US National Research Council has spelled out a paradigm change from in vivo to in vitro testing strategies 
as a vision for the $21^{\text {st }}$ century (CTTAEA and NRC, 2007): "The committee envisions a new toxicity-testing system that evaluates biologically significant perturbations in key toxicity pathways by using new methods in computational biology and a comprehensive array of in vitro tests based on human biology."

As a consequence of this vision, international efforts are striving to accomplish this paradigm change for the safety testing of bulk chemicals. As regards the safety testing of nanomaterials, where validated test methods or testing strategies so far do not exist, scientific and political efforts should set out to develop a non-animal testing strategy from the beginning. At a time, when global incentives strive for a paradigm change in toxicology, it is no longer ethically acceptable to start out by devising testing strategies, which are based on the outdated test methods.

Hazard identification, exposure and risk assessment should be combined to a tiered-testing and assessment strategy making use of non-animal test methods. Experts in the field of non-animal test methods should be called for their advice on the compilation of such non-animal testing strategies to ensure that these meet the state-of-the-art of in vitro and in silico technologies, while at the same time ensuring human health and environmental protection.

Risk assessment is conceived as a key element to protect human dignity in regard to nanotechnological products. This risk assessment should be based upon scientifically valid and ethically acceptable non-animal test methods and testing strategies.

Risk assessment is considered to be a key element to ensuring the protection of human dignity when making use of a new technology, in order to grant humans the fundamental right of respect for physical integrity. Evidently, this includes protection from harm through intended or unintended exposure to man-made substances and products. However this goal can only be met if scientifically reliable and relevant test methods are used for the underlying substance and product hazard assessment. Apart from the above-mentioned general scientific criticism towards animal tests (National Academy of Sciences, 2007), specific animal test methods for nanotoxicological testing have not yet been validated and their use is being based upon "experience" instead. Such methods should be considered inadequate to protect humans - or their dignity. Furthermore, animal test methods pose new ethical problems, which also should not be disregarded in pursuing solutions that aim to protect human dignity.

The recommendation of the European Group of Ethics, to "devise valid animal testing models to monitor the side effects of nanomedical devices" does not address these fundamental problems of current safety testing strategies. Those responsible for human health safety - and the protection of human dignity - should aim to rely on ethically acceptable and scientifically reliable and relevant testing strategies.

European citizens are concerned about animal welfare. Public involvement in the debate on nanotechnology should take into account information on resulting animal research.

12 http://ec.europa.eu/food/animal/welfare/survey/sp_barometer_aw_en.pdf

$13 \mathrm{http}: / /$ ec.europa.eu/public_opinion/archives/ebs/ebs_225_report_en.pdf
In accordance with a Eurobarometer published in March 2007 on the "attitudes of EU citizens on animal welfare"12, EU citizens see animal welfare as a matter of great importance, regardless of social or demographic factors. Likewise, in accordance with a Eurobarometer published in June 2005, on "societal values, science and technology"13, $82 \%$ of the Europeans agree with the statement that humans have a duty to protect the rights of animals whatever the cost.

Animal welfare issues should be included when making available to the public information on the costs of nanotechnology. The public should be informed in which areas this new technology has the potential to lead to an increase in animal testing and how distressful these experiments actually are to the animals. This is more so true when new products are ethically questionable also from human ethical point of view. If the public is hesitant to use the new technologies in the first place, a rise in animal experimentation due to emerging nanotechnologies should be subject to public discussion.

\section{Conclusion}

In order to pay full regard to animal welfare issues, as requested by the Treaty of Lisbon, the emerging research area nanotechnology can and should be used to accelerate the paradigm change called for in toxicological testing - not least because of the new non-animal technological developments it is bringing forward. To achieve this goal, however, the funding for nonanimal test methods requires considerable increase; and funding requirements should be adjusted to specifically promote nonanimal test methods. Since risk assessment is considered to be a prerequisite to protect human dignity, it should no longer be founded on scientifically deficient and at the same time ethically questionable test methods. The animal welfare issues regarding ongoing developments in nanotechnology should be included in all ethical deliberations and public debates regarding nanotechnology to enable European citizens to obtain a comprehensive opinion on the overall "costs" of nanotechnological products.

\section{References}

Note: All websites were accessed in October and November 2009.

Aguar, P. and Nicholas, J. J. M. (2008). EU nanotechnology R \& $\mathrm{D}$ in the field of health and environmental impact of nanoparticles. European Commission, Directorate General Research of 28 January 2008. ftp://ftp.cordis.europa.eu/pub/nanotechnology/docs/final-version.pdf

BMBF (2007). German Federal Ministry of Education and Research-Nanoinitiative - Action Plan 2010. 34pp. http://www. bmbf.de/pub/nano_initiative_action_plan_2010.pdf

BMBF (2009). German Federal Ministry of Education and Research-Nano. DE-report 2009 - Status quo of nanotech- 
nology in Germany. 90pp. http://www.bmbf.de/pub/nanode_ report_2009_en.pdf

Brandenberger, C., Rothen-Rutishauser, B., Mühlfeld, C. et al. (2009). Effects and uptake of gold nanoparticles deposited at the air-liquid interface of a human epithelial airway model. Toxicol. Appl Pharmacol. epub 29 Sept 09.

Commission of the European Communities (2005). Communication from the Commission to the Council, the European Parliament and the European Economic and Social Committee - Nanosciences and nanotechnologies: An action plan for Europe 2005-2009. COM(2005)243 of 7 June 2005. ftp://ftp. cordis.europa.eu/pub/nanotechnology/docs/action_plan_brochure.pdf

Commission of the European Communities (2007). Communication from the Commission to the Council, the European Parliament and the European Economic and Social Committee - Nanosciences and nanotechnologies: an action plan for Europe 2005-2009. First implementation report 2005-2007. COM 2007 (505) final of 6 September 2007. ftp://ftp.cordis. europa.eu/pub/nanotechnology/docs/com_2007_0505_f_ en.pdf

Commission of the European Communities (2008). Commission Recommendation on a code of conduct for responsible nanosciences and nanotechnologies research. C(2008) $424 \mathrm{fi}$ nal of 7 February 2008. http://ec.europa.eu/nanotechnology/ pdf/nanocode-rec_pe0894c_en.pdf

CTTAEA and NRC (2007). Committee on Toxicity Testing and Assessment of Environmental Agents, US National Research Council. Toxicity Testing in the 21st Century: A Vision and a Strategy. National Academics Press, 216 pp. http://www.nap. edu/catalog.php?record_id=11970\#toc

ECETOC (2006). Testing Strategies to Establish the Safety of Nanomaterials - 7-8 November 2005, Barcelona. Workshop Report No. 7. European Centre for Ecotoxicology and Toxicology of Chemicals, Brussels, Belgium.

EGE (2007). Opinion of the European Group on Ethics in Science and New Technologies to the European Commission. Ethical aspects of nanomedicine. Opinion No. 21 of 17 January 2007. http://ec.europa.eu/european_group_ethics/activities/docs/opinion_21_nano_en.pdf

European Commission - European Research Area (2009). Alternative testing strategies - progress report 2009. Commission of the European Communities - Directorate General Research. EM Nogueiro (ed.). 280pp. http://cordis.europa.eu/ documents/documentlibrary/106691831EN6.pdf

Gellein, K., Hoel, S., Evje, L. and Syversen, T. (2009). The colony formation assay as an indicator of carbon nanotube toxicity examined in three cell lines. Nanotoxicol.3(3), 215-221.

Hullmann, A (2008). European activities in the ethical, legal \& social aspects (ELSA) \& governance of nanotechnology. European Commission, DG Research of 1 October 2008. ftp:// ftp.cordis.europa.eu/pub/nanotechnology/docs/elsa_governance_nano.pdf

Lenz, A. G., Karg, E., Lentner, B. et al. (2009) A dose-controlled system for air-liquid interface cell exposure and application to zinc oxide nanoparticles. Part Fibre Toxicol, in press.
National Academy of Sciencies (2007). Toxicity testing in the 21 st century - a vision and a strategy. Report in Brief. 4 pp. http://dels.nas.edu/dels/rpt_briefs/Toxicity_Testing_final.pdf

Oberdörster, G., Maynard, A., Donaldson, K. et al . (2005). Principles for characterizing the potential human health effects from exposure to nanomaterials: elements of a screening strategy. Part. Fibre Toxicol. 2, 8. http://www.ncbi.nlm.nih. gov/pmc/articles/PMC1260029/?tool=pubmed

Sauer, U. G. (2009). Animal and non-animal experiments in nanotechnology - the results of a critical literature survey. $A L$ TEX 26, 109-134.

Sayes, C. M., Reed, K. L. and Warheit, D. B. (2007). Assessing toxicity of fine and nanoparticles: Comparing in vitro measurements to in vivo pulmonary toxicity profiles. Toxicol. Sci. 97, 163-80

SCCP (2007). Opinion of the Scientific Committee for Consumer Products (SCCP) on the "safety of nanomaterials in cosmetic products". SCCP/1147/07 of 18 December 2007. http://ec.europa.eu/health/ph_risk/committees/04_sccp/docs/ sccp_o_123.pdf

SCENIHR (2009). The Scientific Committee on Emerging and Newly Identified Health Risks Opinion on Risk assessment of products of nanotechnologies of 19 January 2009. http:// ec.europa.eu/health/ph_risk/committees/04_scenihr/docs/ scenihr_o_023.pdf

Skrabanek, P. and McCormick, J. (1989). Follies and Fallacies in Medicine. Tarragon Press, Glasgow.

Swiss Federal Council - Schweizer Bundesrat (2008). Aktionsplan Synthetische Nanomaterialien. Bericht des Bundesrates vom 9. April 2008. http://www.bafu.admin.ch/publikationen/ publikation/00574/index.html?lang=de

Treaty of Lisbon (2008). Consolidated versions of the Treaty on European Union and the Treaty on the Functioning of the European Union. Official J. C 115/01, 9 May 2008. http://europa.eu/lisbon_treaty/full_text/index_en.htm

Wahl, B., Daum, N., Ohrem, H. L. and Lehr, C. M. (2008). Novel luminescence assay offers new possibilities for the risk assessment of silica nanoparticles. Nanotoxicol. 2(4), 243-251.

Warheit, D. B., Hoke, R. A., Finlay, C. et al. (2007). Development of a base set of toxicity tests using ultrafine $\mathrm{TiO} 2$ particles as a component of nanoparticle risk assessment. Toxicol. Lett. 171, 99-110.

\section{Acknowledgements}

The financial support by the Foundation AnimalFree Research, Zurich, Switzerland, is gratefully acknowledged.

\section{Correspondence to}

Dr. med. vet. Ursula G. Sauer

Scientific Consultancy - Animal Welfare

Hallstattfeld 16

85579 Neubiberg

Germany

Tel.: +49-89-66090717

e-mail: ursula.sauer@sauerug.de 